\title{
Report of a PMS2 Germline Mutation Patient Presenting with Endometrial and Parotid Cancer
}

\author{
Mariela Rodríguez-Suárez ${ }^{1,2}$, Yaritza Díaz-Algorri ${ }^{7}$, Robert Hunter-Mellado ${ }^{8}$, Luis Ferrer-Torres ${ }^{9}$ and Marcia Cruz-Correa $^{1,2,3,4,5,6^{*}}$ \\ ${ }^{1}$ University of Puerto Rico, Comprehensive Cancer Centre, USA \\ ${ }^{2}$ Department of Internal Medicine, University of Puerto Rico Medical Sciences Campus, USA \\ ${ }^{3}$ Department of Biochemistry, University of Puerto Rico Medical Sciences Campus, USA \\ ${ }^{4}$ Department of Surgery, University of Puerto Rico Medical Sciences Campus, USA \\ ${ }^{5}$ UPR/MDACC Partnership for Excellence in Cancer Research Program, USA \\ ${ }^{6}$ Department of Medicine, Johns Hopkins University, USA \\ ${ }^{7}$ San Juan Bautista School of Medicine, Dean of Graduate Programs, USA \\ ${ }^{8}$ Departments of Internal Medicine and Cancer Research Unit, Universidad Central del Caribe School of Medicine, USA \\ ${ }^{9}$ Hato Rey Pathology Lab, HIMA Caguas, USA \\ *Corresponding author: Correa MC, University of Puerto Rico Comprehensive Cancer Centre, PMB 711 Ave. 89 Ste. 105, San Juan, P.R. 00927-6346, USA, Tel: \\ 1-787-772- 1221; Fax: 1-787-522-3282; E-mail: marcia.cruz1@upr.edu
}

Rec Date: Feb 03, 2016; Acc Date: May 13, 2016; Pub Date: May 18, 2016

Copyright: $\odot 2016$ Correa MC, This is an open-access article distributed under the terms of the Creative Commons Attribution License, which permits unrestricted use, distribution, and reproduction in any medium, provided the original author and source are credited.

\begin{abstract}
Colorectal cancer (CRC) is the second leading cause of cancer among men and women and represents the leading cause of cancer death in Puerto Rico. Familial CRC accounts for $10-15 \%$ of the total CRC cases, with Lynch syndrome (LS) implicated in $2-4 \%$ of cases. Limited information is available on the prevalence, clinical manifestations, and genetic mutations of hereditary CRC in USA Hispanics. In this paper we report a PMS2 mutation in a Puerto Rican Hispanic patient with LS recruited through the Puerto Rico Familial Colorectal Cancer Registry. At the age of 35 years our proband was diagnosed with endometrial cancer with parotid cancer established the following year. A diagnosis of Lynch Syndrome was established through analysis of protein expression by immunohistochemistry and genetic sequencing of mismatch repair genes. Mutation in the PMS2 gene is rarely linked with LS. This case report adds Parotid Carcinoma to the spectrum of malignant conditions associated to LS. We emphasize on the importance of genetic testing in at-risk patients for hereditary CRC from various racial backgrounds, and underscore the need for genetic counselling of patients and their family members. Recognition of LS carriers will allow early detection of malignant condition and the implementation of effective therapy, which will ultimately improve prognosis.
\end{abstract}

Keywords: PMS2 mutation; MLH1; MSH2; MSH6; EPCAM; Lynch syndrome; Hereditary nonpolyposis colorectal cancer; Colorectal cancer; Endometrial cancer; Parotid cancer; Hispanics

\section{Abbreviations:}

PR (Puerto Rico); CRC (Colorectal Cancer); USA (United States of America); LS (Lynch Syndrome); MMR (Mismatch Repair); MSI (Microsatellite Instability); MSI-H (MSI-High); MSI-L (MSI-Low); PCR (Polymerase Chain Reaction); CMMR-D (Constitutional MMRDeficiency); MTS (Muir-Torre Syndrome); IHC (Immunohistochemistry); VUS (Variants of Uncertain Significance); PURIFICAR (Puerto Rico Familial Colorectal Cancer Registry); UPRCCC (University of Puerto Rico Comprehensive Cancer Centre); FAP (Familial Adenomatous Polyposis); Attenuated FAP (AFAP); UPR (University of Puerto Rico); FNA (Fine Needle Aspiration); MEC (Mucoepidermoid Carcinoma); Genomic DNA (GDNA); MLPA (Multiplex Ligation-Dependent Probe Amplification); HNPCC (Hereditary Nonpolyposis Colorectal Cancer)

\section{Introduction}

In Puerto Rico (PR), Colorectal cancer (CRC) is the second leading cause of cancer among men and women [1]. During the last decade, the mortality rate of CRC in PR has remained high and it is the first leading cause of cancer death, accounting for $13.1 \%$ of cancer deaths in men and $13.6 \%$ in women [1]. In the United States (US), CRC is the third leading cause of cancer death in Hispanics, accounting for 16.1 deaths per 100,000 men and 10.7 deaths per 100,000 women [2]. Familial CRC is implicated in $10-15 \%$ of all CRCs [3,4], and several studies suggest that inherited genetic factors have a major influence in the pathogenesis of up to one third of all CRC cases [5]. Lynch syndrome (LS), also known as hereditary nonpolyposis colorectal cancer (HNPCC), is the most common hereditary CRC syndrome and is responsible for approximately $2-4 \%$ of all CRC cases [6,7]. The Amsterdam criteria are a set of diagnostic criteria used by physicians to help identify families that are likely to have LS, which was revised to increase the sensitivity by also including the LS-tumor spectrum and the MSI tumor testing results as part of the criteria $[6,7]$.

LS is a highly penetrant, autosomal dominant cancer-prone syndrome caused by germline mutation in one of the four mismatch repair (MMR) genes: mutL homolog 1 (MLH1), mutS homolog 2 
(MSH2), mutS homolog 6 (MSH6), postmeiotic segregation increased DNA mismatch repair (PMS2), or deletions in epithelial cell adhesion molecule (EPCAM) gene [6,7]. Deletions in the 3' end of EPCAM causes silencing of the neighboring $\mathrm{MSH} 2$ gene by hypermethylation of the promoter resulting in loss of MSH2 expression without detectable MSH2 germline mutation [6]. MMR genes products are responsible for maintaining genomic integrity by correcting nucleotide errors that have escaped the usual editing function of DNA polymerase [6]. This results in genomic microsatellite instability (MSI), an increased mutation rate through the accumulation of DNA polymerase errors [6,8]. Therefore, loss of MMR promotes neoplasia and is associated with a lifetime risk of $40-80 \%$ of malignant transformation $[6,8]$. Target organs at risk for malignancy include CRC and endometrial cancers, followed by ovarian, gastric, small bowel, hepatobiliary tract, pancreas, urinary tract, brain, skin, and cutaneous sebaceous glands [6,9]. A number of unusual sites associated to the mutation have been recently described including breast, prostate, lung, thyroid, adrenal cortex, sarcomas, and melanomas, show MMR deficiency and are being reported in patients with LS $[9,10]$. Nevertheless, parotid cancer has not been reported as part of the spectrum of "unusual tumors" due to absence of clinical evidence to support their inclusion into the LS-cancer spectrum nor to recommend general screening and surveillance guidelines for LS [9].

Studies suggest that there are differences in cancer risks depending on the gene that is mutated, increasing the CRC risk in families diagnosed with MLH1 mutation [6,11]. Cases with MLH1 deficiency are diagnosed at a younger age compared to MSH2 and MSH6, with a median age of 40 years for any cancer and 41 years for CRC [11]. MLH1 and MSH2 mutations often occur in families that fulfill Amsterdam criteria with tumors that are MSI-high (MSI-H). Histologically these neoplasms are characterized by the "presence of tumor infiltrating lymphocytes, Crohn's-like lymphocytic reaction, mucinous/signet-ring differentiation, or medullary growth patterns" [12]. Deficiency of MSH2 is associated with a higher risk of extracolonic tumors [6]. MSH6 mutations have later onset, higher risk of endometrial cancer, and/or MSI-low (MSI-L) $[6,13]$. Information regarding cancer risks for PMS2 gene mutation is limited, but in general the 4 overall cancer risks appear to be lower. [14]. CRC in heterozygous PMS2 patients is located predominantly proximal to the splenic flexure, show MSI, and behave mostly like sporadic CRC with no significant family history of any LS-associated cancer $[13,15]$.

The clinical consequences of PMS2 germline mutations are poorly understood compared with other MMR gene mutations $[7,14]$. This is primarily due to the limited number of carriers described to date and the lower penetrance compared to the other MMR genes [14,15]. PMS2 is a MutL homologue MMR gene located on chromosome 7p22, which interferes with the analysis of such mutations given the presence of a large family of pseudogenes located on the same chromosome, resulting in missed diagnoses of PMS2 mutations [13-15]. There are up to 15 non-functional PMS2-related genes on the long arm resembling the 5' end of PMS2 exons 1-5 and one 98\% identical to the 3' end of exons 9 and 11-15, which is known as PMS2CL and was detected by Nakagawa et al. [15]. However, most of these complications have recently been overcome with long range polymerase chain reaction (PCR) to amplify the PMS2 gene rather than its pseudogenes, thus detection of such mutations is more accurate except for exons 13-15 in which pseudogene-related conversion may confound the analysis $[7,15]$.
In addition to LS, PMS2 mutation is associated with other hereditary cancer predisposition syndromes such as Turcot syndrome and Constitutional MMR-deficiency (CMMR-D) syndrome [16,17]. Previous reports of individuals with biallelic germline PMS2 mutations typically present with malignancy at younger age and have a distinct phenotype from the classic LS, often consisting of gastrointestinal and hematologic malignancies, brain tumors, and features of neurofibromatosis type 1 including café-au5 lait-spots $[14,16]$. Another profile that is suggestive of PMS2 mutation has been recorded in one prior report of Muir-Torre syndrome (MTS) [18], which is a phenotypic variant of LS and comprises approximately 1-3\% of LS families [19,20]. In such study, isolated loss of PMS2 in immunohistochemistry (IHC) staining was recorded in 1 of $12 \mathrm{MMR}$ deficiency cases [18]. The most common associated neoplasms in MTS are CRC, genitourinary tumors, breast carcinomas, and hematologic disorders [19]. Other less common malignancies include those of the parotid gland, larynx, biliary tract, small intestine, lung, paraganglioma, and chondrosarcoma [19].

Variants of uncertain significance (VUS) limit the interpretation of genetic testing results among Hispanics who undergo germline genetic testing, due to the little information available on the prevalence, clinical manifestations, and genetic mutations in the USA Hispanics with LS [21]. Published data suggests that mutations in the PMS2 gene are rare in the etiology of LS. Mutations in MLH1 and MSH2 account for approximately $60-80 \%$ of all LS cases, while PMS2 mutations represent $1-13.7 \%[17,22]$. Furthermore, the majority of families with PMS2 mutations do not comply with the Amsterdam criteria [23]. It is important to understand the clinical manifestations and risks of PMS2 mutations for adequate screening, surveillance, and counseling. Using the PR Familial Colorectal Cancer Registry (PURIFICAR), we identified patients for LS who have had germline genetic testing [24]. The aim of this study was to report the unusual clinicopathological phenotype of a germline PMS2 mutation found in a Caribbean Hispanic patient with LS recruited through PURIFICAR.

\section{Methods}

\section{Recruitment}

The proband was identified through PURIFICAR [24]. Individuals with either personal or family history of possible familial CRC and/or polyposis are referred to PURIFICAR by gastroenterologists, oncologists, and colorectal surgeons [21]. This registry was established in 2006 at the University of PR Comprehensive Cancer Center (UPRCCC) and has received support from the National Institutes of Health, UPRCCC, the PR Gastroenterology Association (http:// www.gastropr.org), and the PR Colorectal Cancer Coalition (http:// cancercolonpr.org) [21]. Subjects enrolled are USA and Caribbean Hispanics with clinical and/or genetic diagnosis of familial adenomatous polyposis (FAP), attenuated FAP (AFAP), hamartomatous polyposis syndrome, or LS [25]. Probands are referred to the University of Puerto Rico (UPR) Cancer Genetics Clinic where they are offered genetic counseling and testing, diagnostic and surveillance endoscopic procedures, referral to specialists, and communication with insurance companies to facilitate approval of services [25]. A baseline questionnaire capturing medical, environmental exposures and cancer family history is completed for each consented proband [25]. Pedigrees for each proband are completed to trace the number of affected relatives with polyposis and/or cancer using PROGENY or INVITAE software [26,27]. 
Medical, surgical and pathology reports are obtained from medical records to confirm cancer diagnosis notified by probands. Affected family members living in PR and USA are also invited to participate in the registry. According to Cruz-Correa et al., in this registry 35 individuals have been diagnosed with LS, consisting of 14 males and 21 females, with a mean age of CRC at 46.3 ( \pm 13.4 ) years and the following distribution of LS mutations: $66.7 \%$ in the MSH2 gene, $25.0 \%$ in MLH1, and $8.3 \%$ in MSH6 [25]. For the current study, analysis was limited to the proband and her family.

\section{IHC analysis}

Commercial IHC analysis for the presence of MLH1, MSH2, MSH6, and PMS2 proteins was evaluated in endometrial and parotid tumor blocks.

\section{Genetic sequence analysis}

Genetic diagnosis of LS was established by identifying mutation with commercial sequence analysis, Ambry Genetics Corp. [28]. DNA was extracted from peripheral blood samples that were sent to Ambry Genetics Corp. for molecular testing. As part of this process, Ambry Genetics Corp. performed full gene deletion/duplication analysis using the PMS2 SALSA multiplex ligation-dependent probe amplification (MLPA) KIT P008-B1, but it cannot accurately assess if deletions/ duplications in exons 13-15 occur in the PMS2 gene or a pseudogene [28]. Thus, if a deletion is detected in exons 13-15, Ambry Genetics Corp. performs double stranded sequencing of the appropriate exon(s) of the pseudogene PMS2CL to determine if the deletion is located in the PMS2 gene or pseudogene [28].

Analytical Range: According to Ambry Genetics Corp., PMS2 exons 1-15 coding domains plus at least 20 bases into the 5' and 3' ends of all the introns and untranslated regions are analyzed by sequencing. Gross deletion/duplication analysis determined gene copy number for exons 1-15 of PMS2 [28].

\section{Results}

\section{Patient history}

Our proband, defined as the first person in the family in which the mutation was detected, is a 36-year-old female with significant medical history of idiopathic thrombocytopenic purpura, endometrial cancer, and parotid cancer. She has no history of alcohol, cigarette smoking, or drug use. She was first diagnosed with endometrial cancer at the age of 35 years (2013) by endometrial biopsy, which determined a welldifferentiated adenocarcinoma, FIGO grade I, nuclear grade 1. IHC studies performed were focally positive for p-16, p-53, KI-67, and positive for betacatenin- A, vimentin, estrogen receptor, and progesterone receptor. She then underwent abdominal hysterectomy and pathologic analysis confirmed endometrial adenocarcinoma with FIGO grade II/III, nuclear grade $3 / 3$, invading less than half of the myometrium. Neither endocervical nor vascular invasion was identified and it was classified as FIGO stage IA. In 2014, at 36 years of age, she developed a painless swelling near her right ear and was referred for sonographically-guided fine needle aspiration (FNA) of the right parotid gland. FNA was suspicious for low-grade mucoepidermoid carcinoma (MEC) and the differential diagnosis also included a mucus retention cyst or mucocele. CT scan of the neck revealed a small right parotid gland nodular lesion and scattered discrete lymph nodes on the right jugulo-digastric space and left submandibular region; excision was suggested to exclude malignancy. Subsequently she underwent right parotidectomy with lymph node biopsy. Pathologic analysis confirmed low-grade MEC measuring 1.0 $\mathrm{cm}$ without lymphatic, vascular, and perineural invasions. She was then referred by her oncologist to the UPR Cancer Genetics Clinic after the endometrial tumor revealed absence of PMS2 expression on IHC assays for MMR proteins. Surveillance esophagogastroduodenoscopies and colonoscopies have been unremarkable, asides from hiatal hernia and hemorrhoids. The proband's family history was also positive for cancer in her paternal side: CRC in her father's uncle, endometrial cancer in her father's cousin, breast cancer in her father's cousin and father's aunt, CRC and ureteral cancer in her grandfather. A detailed pedigree is shown in Figure 1.

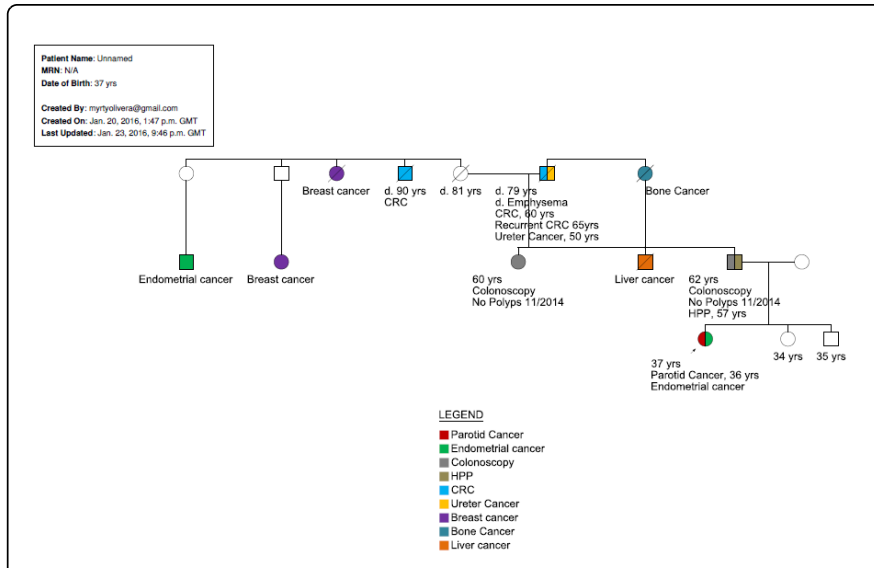

Figure 1: A detailed pedigree.

Our proband's family pedigree does not comply with Amsterdam criteria. It shows five family members with history of cancer in her paternal side. In the first generation family line, the proband's paternal grandfather was diagnosed with CRC and ureter cancer, her father's maternal uncle with CRC, and her father's maternal aunt with breast cancer. In the second generation, one of her father's maternal cousins was diagnosed with breast cancer and another one with endometrial cancer. In the third generation, the proband was diagnosed with endometrial and parotid cancers. A full detailed pedigree is shown in Figure 1.

IHC and Genetic analysis: Commercial IHC analysis of the proband's endometrial tumor expressed MLH1, MSH2 and MSH6 proteins, with absence of PMS2 protein expression, as shown in Figure 2. Genetic sequence analysis done by Ambry Genetics Corp. detected that the proband is heterozygous for the p.S461 pathogenic mutation located in exon 2 of PMS2. This alteration is caused by a G>T change at the nucleotide 137 (c.137G $>$ T). This single nucleotide substitution leads to a missense mutation, where the serine at codon 46 is replaced by isoleucine, an amino acid with differing properties [28]. Since parotid malignancy has been reported in MTS [19], we considered the proband's parotid MEC as one of the "unusual LS tumors" and we ordered IHC staining for all MMR proteins. However, her parotid tumor IHC staining was positive for all MMR proteins, as shown in Figure 3. 


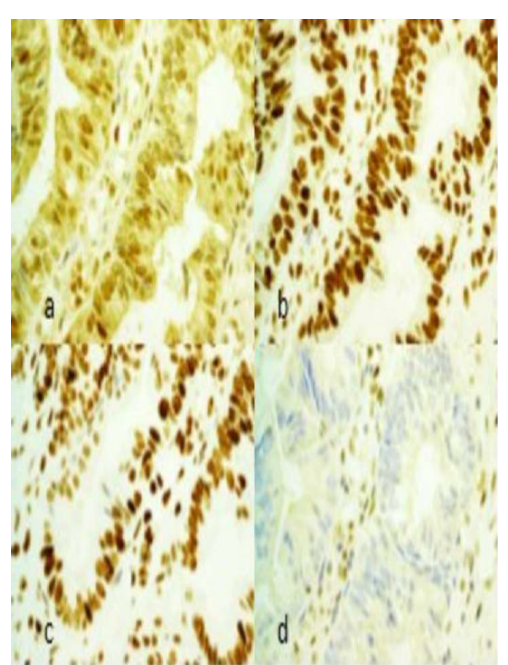

Figure 2: Endometroid adenocarcinoma, H\&E 400x. a,b,c: MLH1, MLH2, and MSH6 proteins by immunohistochemistry technique (DAB) expressed in the tumor and stroma d: PMS2 protein is not expressed by the tumor (stroma as an internal control is positive) Technique for detecting MSI proteins: Primary antibodies and detection system by DAKO, Carpinteria, California, USA.

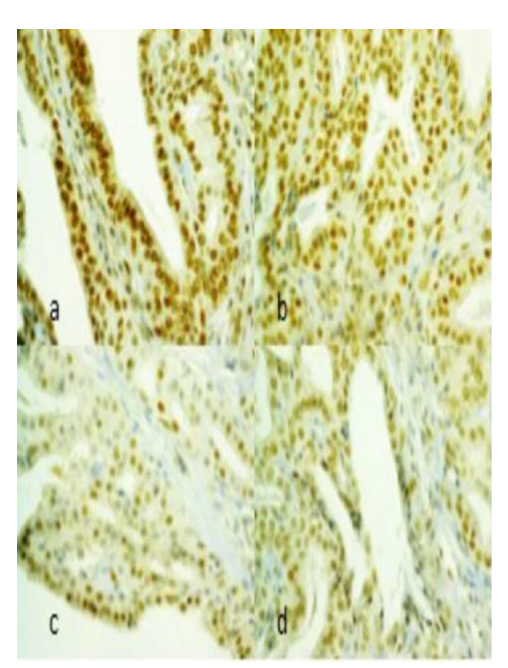

Figure 3: Mucoepidermoid carcinoma, low grade, H\&E 400x. a,b,c,d: MLH1, MLH2, MSH6, and PMS2 proteins by immunohistochemistry technique (DAB) expressed in the tumor and stroma.

\section{Discussion}

LS is a highly penetrant syndrome that is inherited in an autosomal dominant pattern and affects less than $5 \%$ of all CRC cases [6]. It is associated with germline mutations in MMR genes, which increases risk for CRC and endometrial cancer, followed by gastric, ovarian, urinary, sebaceous glands, and brain cancers [6,9]. The Amsterdam criteria has been used to identify families with this inherited syndrome, however it has limited sensitivity and specificity since $40 \%$ of families with MMR gene mutation do not meet this criteria and approximately $50 \%$ of families who meet the Amsterdam criteria do not have detectable defect in the MMR genes [6]. Therefore, various physicians have been screening CRC and endometrial tumors for LS at the time of surgical diagnosis, often using IHC to assess the status of MMR proteins, which allows for the identification of individuals at increased risk regardless of their age or family history and facilitates the search for mutations by indicating the most likely mutated gene based on the absence of its protein in the tumor [14]. So far, the most reliable way to diagnose LS is to detect a mutation in the MMR genes of the suspected patient [2], most of them in the MLH1 and MSH2 genes [29]. The most common MMR gene affected in non-Hispanic populations is MLH1 [29]. In contrast, the mutation spectrum in Caribbean Hispanics portrayed by our group is composed mostly of MSH2, followed by MLH1 and MSH6 [25]. Also, our group described a novel MLH1 gene mutation c.2044_2045del found in one Hispanic family from PR [21].

Our familial cancer registry, PURIFICAR, contains 35 Hispanic families with MMR mutations or MMR protein deficient tumors. Through this registry we identified our proband, a 36-year-old female diagnosed with endometrial cancer at age 35 and parotid cancer at age 36. Her family history presents with cancers highly associated with LS such as CRC, ureteral cancer, and endometrial carcinoma (Figure 1). Upon the evaluation of the proband's MMR sequence analysis, we found the individual is heterozygous for the p.S461 mutation in the PMS2 gene. Because the development of improved PMS2 mutation diagnostic measures is recent, clinical reports concerning heterozygous PMS2 mutation carriers include small cohorts [7]. Previous studies described a lower PMS2 mutation penetrance for CRC and endometrial cancer compared with MLH1 and MSH2 mutation carriers, but similar or lower risks when compared with MSH6 mutation carriers [7]. Moreover, relatives of biallelic PMS2 mutation carriers rarely develop CRC or other LS-related cancers [7]. It has been hypothesized regarding this lower penetrance of PMS2 mutations that MLH1 can form a heterodimer with MLH3 or PMS1 in the absence of functional PMS2; therefore, these MLH1/MLH3 and/or MLH1/PMS1 heterodimers may partially compensate for the MutLa heterodimer between MLH1 and PMS2 in the MMR process $[7,14]$.

Studies focused on the phenotype of LS due to germline PMS2 mutation agreed that the cumulative cancer risk to age 70 for CRC is $15-20 \%$, for endometrial cancer is $15-20 \%$, and for any LS-associated cancer is $25-32 \%[7,14]$. Senter et al. reported that the incidence of CRC in PMS2 mutation carriers was 5.2 fold higher than the general population, the incidence of endometrial cancer was 7.5 fold higher, and there was no elevated risk for non-LS-associated cancers [14]. Regarding tumor MSI, Hendriks et al. identified seven PMS2 mutations, including four genomic rearrangements and three point mutations, and all LS-associated tumors showed MSI-H [23]. Another profile that has recently been associated with the loss of PMS2 or MSH6 expression is MTS $[18,30]$. MTS is identified by the lack of MLH1 and/or MSH2 in IHC of tissue samples, but more recently, absence of MSH6 and/or PMS2 has been shown in sebaceous tumors of these patients [31]. Orta and co-workers reported loss of PMS2 unaccompanied by MLH1 loss [18]. From a total of 12 patients, IHC staining included concurrent loss of MSH2/MSH6 in 8 patients (67\%), concurrent loss of MLH1/PMS2 in 2 patients (17\%), isolated loss of MSH6 in one patient (8\%), and isolated loss of PMS2 in one patient (8\%) [18]. Such study recommended that since isolated loss and concurrent loss of MSH6 or PMS2 can occur in sebaceous neoplasms and CRC, the antibody panel should include MSH6 and PMS2 [18]. 
Besides the abovementioned LS-associated cancers, limited information regarding parotid cancer as part of the LS-tumor spectrum is known. However, it can be seen in MTS, which is considered a subtype of LS [19]. Our proband was diagnosed with lowgrade MEC of the parotid gland, the most common malignant salivary tumor [32,33]. Cancer of the salivary glands is rare and usually develops in the parotid glands, of which about $20 \%$ are malignant $[33,34]$. MEC is most commonly seen between the ages of 35-65 years [34]. It is composed of mucin-producing cells, clear cells and squamoid cells, and expresses various membrane-bound mucins MUC1, MUC4, MUC5AC, and MUC5B [32,34]. It is also characterized by a specific translocation $\mathrm{t}(11 ; 19)$ (q14-21;p12-13), which creates a novel fusion product MECT1-MAML2 and disrupts the Notch signaling pathway that plays a role in the normal development of many tissues and cell types [34]. So far, IHC analysis for MMR protein expression in salivary gland tumors is not usually performed $[35,36]$. Castrilli et al. examined the expression of MLH1 and MSH2 by IHC in salivary carcinomas and all carcinomas expressed these proteins, thereby suggesting no MMR defect in the pathogenesis of malignant salivary gland tumors [36]. Our proband's MEC expressed all MMR proteins on IHC despite having PMS2 gene mutation, which could be due to certain degree of residual protein function or the possibility of a coincidental sporadic tumor. According to Martinez and Kolodner, although many missense mutations in MMR genes are lossof- function, various mutations have either no effect on MMR or cause weak MMR defects emphasizing the need for functional testing before determining missense mutations are pathogenic [37].

To date, little is known about the phenotype and cancer risks of PMS2 mutation carriers [7,14]. Furthermore, these individuals do not fulfill the clinical Amsterdam criteria for LS [23], making it harder to identify such mutations. Our report of a patient with PMS2 mutation c. $137 \mathrm{G}>\mathrm{T}$ diagnosed with endometrial and then parotid malignancies contributes to the unusual phenotypic characterization of PMS2 mutation carriers with LS. Previous cases of c.137G $>$ T mutation in patients with CMMR-D syndrome have classified the mutation as pathogenic [17]. Herkert et al. described such mutation in a Dutch family with CMMR-D syndrome, which affected a female proband at the age of 19 years and her younger brother at the age of 15, both developed multiple high-grade dysplastic colon adenomas and jejunal adenocarcinoma; she died at 23 years of age and he died at 22 [38]. The familial cancers included: bladder transitional cell carcinoma and rectal cancer in the female proband, duodenal adenocarcinoma and Tcell acute lymphoid leukemia in her younger brother, CRC in their sister and paternal grandmother, gastric cancer in their maternal grandfather, and endometrial cancer in their maternal grandmother [38]. Borras et al. also described this mutation in a series of Spanish patients with LS [17]. In their study, PMS2 pathogenic mutations accounted for $6 \%$ of the LS patients, where c.137G $>$ T mutation was identified in a family with the male proband affected by CRC and skin tumors at age 60 and his relative affected by bladder cancer [17]. Borras et al. showed the distinct expressivity of PMS2 mutations, where $44 \%$ of families detected with PMS2 pathogenic mutations did not meet any clinical LS criteria [17]. Our report highlights the importance of genetic testing in the Hispanic community in order to identify families with LS. Information about the clinicopathological significance of PMS2 mutations is crucial for appropriate counselling and surveillance of patients and their family members, which could overall help in the patient's prognosis and management.

\section{Core Tip}

Limited information is available about the prevalence, clinical manifestations, and genetic mutations in USA Hispanics with hereditary CRC. LS have been described in less than 5\% of CRC cases. This case report presents the unusual phenotype of a Hispanic patient from PR with a PMS2 gene mutation, which is rare in the etiology of LS. Our objective is to present this uncommon genetic mutation and its clinical manifestations to improve the surveillance and genetic counseling in the medical community, which could significantly influence the patient's prognosis and management.

\section{Author's Contribution}

Mariela C. Rodríguez-Suárez drafted the paper and acquired subject clinical data; Yaritza Díaz-Algorri and Marcia Cruz-Correa designed the study, analyzed the data, and edited the paper; Robert HunterMellado acquired subject clinical data and edited the paper; Luis Ferrer-Torres performed the IHC analysis.

\section{Acknowledgement}

This study was supported by National Institute on Minority Health Disparities Award Number 8U54MD007587-03, 8G12MD007583 and U54MD007587; National Cancer Institute Award Number 5K22CA115913-03, R21CA167220-01, and U54CA096297; Center for Collaborative Research in Health Disparities RCMI Award Number G12MD007600. The authors would like to thank Ambry Genetics Laboratory for their support. The authors are grateful to the family members for their support and cooperation during this study.

\section{References}

1. Tortolero-Luna G, Zi ZD, Pérez-Ríos N, Torres-Cintrón CR, Ortiz-Ortiz KJ, et al. (2006-2010). 201, Puerto Rico Central Cancer Registry: San Juan, Puerto Rico.

2. Gupta S, Ashfaq R, Kapur P, Afonso BB, Nguyen TP, et al. (2010) Microsatellite instability among individuals of Hispanic origin with colorectal cancer. Cancer 116: 4965-4972.

3. Burt RW (2000) Colon cancer screening. Gastroenterology 119: 837-853.

4. Vasen HF, Möslein G, Alonso A, Aretz S, Bernstein I, et al. (2010) Recommendations to improve identification of hereditary and familial colorectal cancer in Europe. Fam Cancer 9: 109-115.

5. Lichtenstein P, Holm NV, Verkasalo PK, Iliadou A, Kaprio J, et al. (2000) Environmental and heritable factors in the causation of cancer--analyses of cohorts of twins from Sweden, Denmark, and Finland. N Engl J Med 343: 78-85.

6. Kastrinos F, Stoffel EM (2014) History, genetics, and strategies for cancer prevention in Lynch syndrome. Clin Gastroenterol Hepatol 12: 715-727.

7. Ten Broeke SW, Brohet RM, Tops CM, van der Klift HM, Velthuizen ME, et al. (2015) Lynch syndrome caused by germline PMS2 mutations: delineating the cancer risk. J Clin Oncol 33: 319-325.

8. Ramsoekh D, Van Leerdam ME, Wagner A, Kuipers EJ (2007) Review article: Detection and management of hereditary non-polyposis colorectal cancer (Lynch syndrome). Aliment Pharmacol Ther 26 Suppl 2: 101-111.

9. Shia J, Holck S, Depetris G, Greenson JK, Klimstra DS (2013) Lynch syndrome-associated neoplasms: a discussion on histopathology and immunohistochemistry. Fam Cancer 12: 241-260.

10. Karamurzin Y, Zeng Z, Stadler ZK, Zhang L, Ouansafi I, et al. (2012) Unusual DNA mismatch repair-deficient tumors in Lynch syndrome: a report of new cases and review of the literature. Hum Pathol 43: 1677-1687. 
Citation: Suárez MR, Algorri YD, Mellado RH, Torres LF, Correa MC (2016) Report of a PMS2 Germline Mutation Patient Presenting with Endometrial and Parotid Cancer. Hereditary Genet 5: 168. doi:10.4172/2161-1041.1000168

Page 6 of 6

11. Goecke T, Schulmann K, Engel C, Holinski-Feder E, Pagenstecher C, et al. (2006) Genotype-phenotype comparison of German MLH1 and MSH2 mutation carriers clinically affected with Lynch syndrome: a report by the German HNPCC Consortium. J Clin Oncol 24: 4285-4292.

12. Umar A, Boland CR, Terdiman JP, Syngal S, de la Chapelle A, et al. (2004) Revised Bethesda guidelines for hereditary nonpolyposis colorectal cancer (Lynch syndrome) and microsatellite instability. J Natl Cancer Inst 96: 261-8.

13. Abdel-Rahman WM, Mecklin JP, Peltomäki P (2006) The genetics of HNPCC: application to diagnosis and screening. Crit Rev Oncol Hematol 58: 208-220.

14. Senter L, Clendenning M, Sotamaa K, Hampel H, Green J, et al. (2008) The clinical phenotype of Lynch syndrome due to germ-line PMS2 mutations. Gastroenterology 135: 419-428.

15. Nakagawa H, Lockman JC, Frankel WL, Hampel H, Steenblock K, et al. (2004) Mismatch repair gene PMS2: disease-causing germline mutations are frequent in patients whose tumors stain negative for PMS2 protein, but paralogous genes obscure mutation detection and interpretation. Cancer Res 64: 4721-4727.

16. Vasen HF, Ghorbanoghli Z, Bourdeaut F, Cabaret O, Caron O, et al. (2014) Guidelines for surveillance of individuals with constitutional mismatch repair-deficiency proposed by the European Consortium "Care for CMMR-D" (C4CMMR-D). J Med Genet 51: 283-293.

17. Borras E, Pineda M, Cadiñanos J, Del Valle J, Brieger A, et al. (2013) Refining the role of PMS2 in Lynch syndrome: germline mutational analysis improved by comprehensive assessment of variants. J Med Genet 50: 552-563.

18. Orta L, Klimstra DS, Qin J, Mecca P, Tang LH, et al. (2009) Towards identification of hereditary DNA mismatch repair deficiency: sebaceous neoplasm warrants routine immunohistochemical screening regardless of patient's age or other clinical characteristics. Am J Surg Pathol 33: 934-944.

19. Eisen DB, Michael DJ (2009) Sebaceous lesions and their associated syndromes: part II. J Am Acad Dermatol 61: 563-578.

20. Shalin SC, Lyle S, Calonje E, Alexander J, Lazar F (2010) Sebaceous neoplasia and the Muir-Torre syndrome: important connections with clinical implications. Histopathology 56: 133-147.

21. Marqués-Lespier JM, Diaz-Algorri Y, Gonzalez-Pons M, Cruz-Correa M (2014) Report of a novel mutation in MLH1 gene in a Hispanic family from Puerto Rico fulfilling classic Amsterdam criteria for Lynch Syndrome. Gastroenterol Res Pract 2014: 527946.

22. Tomsic J, Senter L, Liyanarachchi S, Clendenning M, Vaughn CP, et al. (2013) Recurrent and founder mutations in the PMS2 gene. Clin Genet 83: 238-243.

23. Hendriks YM, Jagmohan-Changur S, van der Klift HM, Morreau H, van Puijenbroek M, et al. (2006) Heterozygous mutations in PMS2 cause hereditary nonpolyposis colorectal carcinoma (Lynch syndrome). Gastroenterology 130: 312-322.

24. Puerto Rico Familiar Colorectal Cancer Registry (PURIFICAR). 2006 Available from: http://purificar.rcm.upr.edu/index_eng.html.

25. Cruz-Correa M, Diaz-Algorri Y, Pérez-Mayoral J, Suleiman-Suleiman W, Gonzalez-Pons Mdel M, et al. (2015) Clinical characterization and mutation spectrum in Caribbean Hispanic families with Lynch syndrome. Fam Cancer 14: 415-425.

26. Progeny, Progeny Software, LLC. 2002.

27. Invitae, Software. (2013).

28. Ambry Genetics Corp. (2014-2015); Available from: http:// www.ambrygen.com.

29. Peltomäki P, Gao X, Mecklin JP (2001) Genotype and phenotype in hereditary nonpolyposis colon cancer: a study of families with different vs. shared predisposing mutations. Fam Cancer 1: 9-15.

30. Chhibber V, Dresser K, Mahalingam M (2008) MSH-6: extending the reliability of immunohistochemistry as a screening tool in Muir-Torre syndrome. Mod Pathol 21: 159-164.

31. Lee BA, Yu L, Ma L, Lind AC, Lu D (2012) Sebaceous neoplasms with mismatch repair protein expressions and the frequency of co-existing visceral tumors. J Am Acad Dermatol 67: 1228-1234.

32. Bai S, Clubwala R, Adler E, Sarta C, Schiff B, et al. (2013) Salivary mucoepidermoid carcinoma: a multi-institutional review of 76 patients. Head Neck Pathol 7: 105-112.

33. Speight PM, Barrett AW (2002) Salivary gland tumours. Oral Dis 8: 229-240.

34. Namboodiripad PC (2014) A review: Immunological markers for malignant salivary gland tumors. J Oral Biol Craniofac Res 4: 127-134.

35. Nagao T, Sato E, Inoue R, Oshiro H, Takahashi RH, et al. (2012) Immunohistochemical analysis of salivary gland tumors: application for surgical pathology practice. Acta Histochem Cytochem 45: 269-282.

36. Castrilli G, Fabiano A, La Torre G, Marigo L, Piantelli C, et al. (2002) Expression of hMSH2 and hMLH1 proteins of the human DNA mismatch repair system in salivary gland tumors. J Oral Pathol Med 31: 234-238

37. Martinez SL, Kolodner RD (2010) Functional analysis of human mismatch repair gene mutations identifies weak alleles and polymorphisms capable of polygenic interactions. Proc Natl Acad Sci USA 107: 5070-5075.

38. Herkert JC, Niessen RC, Olderode-Berends MJ, Veenstra-Knol HE, Vos YJ, et al. (2011) Paediatric intestinal cancer and polyposis due to bi-allelic PMS2 mutations: case series, review and follow-up guidelines. Eur J Cancer 47: 965-982. 